\title{
Applying Two Different Methods to Extend Restricted Dissimilarity Functions
}

\author{
Eduardo Palmeira ${ }^{1}$ Benjamín Bedregal $^{2}$ Humberto Bustince ${ }^{3}$
}

\author{
${ }^{1}$ Depto. de Ciências Exatas e Tecnológicas, UESC, Ilhéus, Brazil, espalmeira@uesc.br. \\ ${ }^{2}$ Depto. de Informática e Matemática Aplicada, UFRN, Natal, Brazil, bedregal@dimap.ufrn.br. \\ ${ }^{3}$ Departamento de Automatica y Computacion, Upna, Pamplona, Spain, bustince@unavarra.es.
}

\begin{abstract}
Based on previous investigations, we have proposed two different methods to extend lattice-valued fuzzy connectives (t-norms, t-conorms, negations and implications) and other related operators, considering a generalized notion of sublattices. Taking into account the obtained results that we have obtained and seeking to analyze the behavior of both extension methods in face of fuzzy operators related to image processing, we have applied these methods so as to extend restricted dissimilarity functions.
\end{abstract}

Keywords: Extension, Restricted Dissimilarity Functions, retractions, e-operators

\section{Introduction}

Consider the following issue: by assuming that $M$ is a sublattice of $L$ and that $T$ is a fuzzy connective (a t-norm, a t-conorm, fuzzy negation, fuzzy implication, etc.) defined on $M$; how can we extend $T$ to $L$ preserving its properties? Our studies in recent years on this issue led us to provide two different extension methods based on a relaxed notion of sublattice. In [30], we presented an extension method via retractions (shortly EMR) that aimed to obtain a minimum extension of a given operator and was able to preserve its properties; yet, results have shown that some properties of fuzzy connectives are not preserved by this method. In order to fix this weakness, we have proposed in [32] another extension method that uses a special function named e-operator. Unlike EMR, the extension method via e-operators (shortly EMEP) is able to preserve most of the properties of extended operators. However, the operator generated from the extension is not minimal, in general.

Now we would like to analyze the behavior of both methods (EMR and EMEP) with respect to the operators related to image processing. In this framework, a very interesting issue is to determine an appropriate way to make global comparison of images. One of the first attempts in this direction was made by Fodor and Roubens' equivalence functions (see [22]). These functions defined which standards should be observed when such a comparisons are made. According to the literature, there are other papers addressing functions aimed at comparing images. For instance, Bustince et al. presented in [11] a particular version of Fodor and Roubens' equivalence functions, named restricted equivalence functions (shortly REF), which are valued in $[0,1]$ and take into account other important criteria on the comparison of images.

Nowadays, some researchers have extended image processing related concepts to the setting of lattices in order for obtaining a much more general framework than $[0,1]$. Such generalization is natural since intensities in color images can be considered as taking values in such lattices.

In this paper we apply EMR and EMEP methods to extend lattice-valued restricted equivalence functions, restricted dissimilarity functions and $E_{e, N^{-}}$ normal functions .

We start by recalling elementary concepts about lattice theory and defining other essential ones in Section 2. In Section 3 we present the extension methods, whereas, Section 4 is devoted to address the extension of restricted dissimilarity functions. Conclusion and some remarks are discussed in Section 5 .

\section{Preliminaries}

\subsection{Bounded Lattices and its Morphisms}

Most definitions and results in this subsection are well known from the literature, so we just present here a clear formalization of them. Moreover, some other key concepts such as $(r, s)$-sublattices are defined. For a deeper reading about these concepts we recommend [9, 17, 21, 23, 24, 25, 30].

Definition 2.1 Let $L$ be a nonempty set. If $\wedge_{L}$ and $\vee_{L}$ are two binary operations on $L$, then $\left\langle L, \wedge_{L}, \vee_{L}\right\rangle$ is a bounded lattice provided that for each $x, y, z \in$ $L$, the following properties hold:

1. $x \wedge_{L} y=y \wedge_{L} x$ and $x \vee_{L} y=y \vee_{L} x$ (symmetry);

2. $\left(x \wedge_{L} y\right) \wedge_{L} z=x \wedge_{L}\left(y \wedge_{L} z\right)$ and $\left(x \vee_{L} y\right) \vee_{L} z=$ $x \vee_{L}\left(y \vee_{L} z\right)$ (associativity);

3. $x \wedge_{L}\left(x \vee_{L} y\right)=x$ and $x \vee_{L}\left(x \wedge_{L} y\right)=x(a b$ sorption law);

4. There are elements $0_{L}$ (bottom) and $1_{L}$ (top) of $L$ such that $x \vee_{L} 0_{L}=x$ and $x \wedge_{L} 1_{L}=x$, for each $x \in L$. 
Moreover, $L$ is called complete whenever each subset of $L$ has a supremum and an infimum element.

It is also possible to establish a partial order on $L$ by relation

$$
x \leqslant_{L} y \text { if and only if } x \wedge_{L} y=x
$$

Remark 1 When $\leqslant_{L}$ is a partial order on $L$ and there are two elements $x$ and $y$ belonging to $L$ such that neither $x \leqslant_{L} y$ nor $y \leqslant_{L} x$, these elements are said to be incomparable and we denote this by $x \| y$. Otherwise we say that these elements are comparable (notation: $x \smile y$ ).

Definition 2.2 Let $\left(L, \wedge_{L}, \vee_{L}, 0_{L}, 1_{L}\right) \quad$ and $\left(M, \wedge_{M}, \vee_{M}, 0_{M}, 1_{M}\right)$ be bounded lattices. A mapping $f: L \longrightarrow M$ is said to be a lattice homomorphism if, for all $x, y \in L$, we have

1. $f\left(x \wedge_{L} y\right)=f(x) \wedge_{M} f(y)$;

2. $f\left(x \vee_{L} y\right)=f(x) \vee_{M} f(y)$;

3. $f\left(0_{L}\right)=0_{M}$ and $f\left(1_{L}\right)=1_{M}$.

Definition 2.3 A given lattice homomorphism $f$ on $L$ is called:

1. A monomorphism if it is injective;

2. An epimorphism if $f$ is surjective;

3. An isomorphism when $f$ is bijective. An automorphism is an isomorphism from a lattice to itself.

Proposition 2.4 [32] Every lattice homomorphism preserves the order.

Proposition 2.5 [32] Let $L$ be a bounded lattice. Then a function $f: L \longrightarrow L$ is an L-automorphism if and only if

1. $f$ is bijective and

2. $x \leqslant_{L} y$ if and only if $f(x) \leqslant_{L} f(y)$.

Definition 2.6 Let $L$ be a bounded lattice and $L^{n}$ the Cartesian product of $n$ copies of $L$. Given a function $f: L^{n} \rightarrow L$, the action of an L-automorphism $\rho$ over $f$ results in the function $f^{\rho}: L^{n} \rightarrow L$ defined as

$$
f^{\rho}\left(x_{1}, \ldots, x_{n}\right)=\rho^{-1}\left(f\left(\rho\left(x_{1}\right), \ldots, \rho\left(x_{n}\right)\right)\right)
$$

In this case, $f^{\rho}$ is said to be a conjugate of $f$.

Remark 2 From now on, lattice homomorphisms will be called just homomorphisms for simplicity. In case we use the concept of homomorphism applied to other objects, the distinction will be made.

\subsection{Retracts and sublattices}

Classically, every subset of an arbitrary lattice $\left\langle L, \wedge_{L}, \vee_{L}\right\rangle$ that is closed for the operations $\wedge_{L}$ and $\vee_{L}$ is called a sublattice. Formally,

Definition 2.7 [9] A (ordinary) sublattice of a lattice $L$ is a subset $M$ of $L$ such that $x, y \in M$ imply $x \wedge_{L} y \in M$ and $x \vee_{L} y \in M$.

In this work, we would like to work in a more flexible framework of sublattice where $M$ needs not be a subset of $L$. Focusing on this idea, we define a generalized notion of sublattice using retractions.

Definition 2.8 A homomorphism $r$ of a lattice $L$ onto a lattice $M$ is said to be a retraction if there exists a homomorphism $s$ of $M$ into $L$ which satisfies $r \circ s=i d_{M}$. A lattice $M$ is called a retract of a lattice $L$ if there is a retraction $r$, of $L$ onto $M$, and $s$ is then called a pseudo-inverse of $r$.

Definition 2.9 Let $L$ and $M$ be arbitrary bounded lattices. We say that $M$ is a $(r, s)$-sublattice of $L$ if $M$ is a retract of $L$ (i.e. $M$ is a sublattice of $L$ up to isomorphisms). In other words, $M$ is a $(r, s)$ sublattice of $L$ if there is a retraction $r$ of $L$ onto $M$ with pseudo-inverse $s: M \rightarrow L$.

Notice that saying that $M$ is a $(r, s)$-sublattice of $L$ means that it is possible to identify $M$, via a homomorphism $s$, with a subset $K=s(M)$ of $L$ that is algebraically invariant, i.e. it preserves the lattice structure of $M$. In other words, $M$ and $K$ are isomorphic (since $\left.r\right|_{K}$ is an isomorphism), hence $K$ works as an algebraic copy of $M$ embedded into $L$.

Definition 2.10 Every retraction $r: L \longrightarrow M$ (with pseudo-inverse $s$ ) that satisfies $s \circ r \leqslant i d_{L}{ }^{1}$ $\left(i d_{L} \leqslant s \circ r\right)$ is called a lower (an upper) retraction. In this case, $M$ is a lower (an upper) retract of $L$.

Definition 2.11 Let $M$ be a $\left(r_{1}, s\right)$-sublattice of $L$. We say that

1. $M$ is a lower $\left(r_{1}, s\right)$-sublattice of $L$ if $r_{1}$ is a lower retraction. Notation: $M<L$ with respect to $\left(r_{1}, s\right)$;

2. $M$ is an upper $\left(r_{1}, s\right)$-sublattice of $L$ whenever $r_{1}$ is an upper retraction. Notation: $M>L$ with respect to $\left(r_{1}, s\right)$;

3. If $r_{1}$ is a lower retraction and there is an upper retraction $r_{2}: L \longrightarrow M$ such that its pseudo-inverse is also $s$, then $M$ is called a full $\left(r_{1}, r_{2}, s\right)$-sublattice of $L$. Notation: $M \unlhd L$ with respect to $\left(r_{1}, r_{2}, s\right)$.

An immediate consequence of the definition of lower (upper) retraction is that, if $M \unlhd L$ then it follows that $s \circ r_{1} \leqslant i d_{L} \leqslant s \circ r_{2}$.

\footnotetext{
${ }^{1}$ If $f$ and $g$ are functions on a lattice $L$ it is said that $f \leqslant g$ if and only if $f(x) \leqslant L g(x)$ for all $x \in L$.
} 


\subsection{Negations on $L$}

A natural extension of the notion of fuzzy negation can be make by considering arbitrary bounded lattices as possible sets of truth values.

Definition 2.12 A mapping $N: L \rightarrow L$ is a negation on $L$ or just an $L$-negation, if the following properties are satisfied for each $x, y \in L$ :

(N1) $N\left(0_{L}\right)=1_{L}$ and $N\left(1_{L}\right)=0_{L}$ and

(N2) If $x \leqslant_{L} y$ then $N(y) \leqslant_{L} N(x)$.

Moreover, the L-negation $N$ is considered strong if it also satisfies the involution property, i.e.

(N3) $N(N(x))=x$ for each $x \in L$.

The L-negation is strict if satisfies the property:

(N4) $N(x)<_{L} N(y)$ whenever $y<_{L} x$.

The L-negation $N$ is frontier if satisfies the property:

(N5) $N(x) \in\left\{0_{L}, 1_{L}\right\}$ if and only if $x=0_{L}$ or $x=1_{L}$.

Remark 3 Note that the definition of strict $L$ negation does not fully recover the usual definition of strict negation (when $L=[0,1]$ ) since in the latter case continuity is also imposed. In this way, if $L=[0,1]$ then the mapping

$$
N(x)=\left\{\begin{aligned}
0 & \text { if } x=1 \\
1 & \text { if } x=0 \\
\frac{1-x}{4} & \text { otherwise. }
\end{aligned}\right.
$$

Remark 4 One can easily verify that for each strong L-negation we have that $x \| y$ if and only if $N(x) \| N(y)$. Notice that although every lattice admits a negation $N$, it is not true that every lattice admits an involutive negation (see Example 2.15).

Example 2.13 Let $L$ be any lattice such that there exists $x_{0} \in L$ with $x_{0} \neq 0_{L}, 1_{L}$. Then the mapping:

$$
N(x)= \begin{cases}0_{L} & \text { if } x=1_{L} \\ 1_{L} & \text { if } x=0_{L} \\ x_{0} & \text { otherwise. }\end{cases}
$$

is a frontier negation. Notice that this example proves that for every lattice $L$ it is possible to define a frontier negation.

An element $e \in L$ is an equilibrium point of an $L$ negation $N$ if $N(e)=e$. Unlike the usual case and the interval-valued case (see [4]), though, strong $L$ negations need not have an equilibrium point, or, in case it exists, the equilibrium point needs not be unique.

Example 2.14 Let $L$ and $M$ be bounded lattices as shown in the Figure 1. The function $N_{1}: M \rightarrow M$ defined by $N_{1}\left(0_{M}\right)=1_{M}, N_{1}(x)=y, N_{1}(y)=x$ and $N_{1}\left(1_{M}\right)=0_{M}$ is a strong $M$-negation. Nevertheless, $N_{1}$ has no equilibrium point.

Now, consider a function $N_{2}: L \rightarrow L$ given by $N_{2}\left(0_{L}\right)=1_{L}, N_{2}(a)=e, N_{2}(e)=a, N_{2}\left(1_{L}\right)=0_{L}$ and $N_{2}(u)=u$ for each $u \in L \backslash\left\{a, e, 0_{L}, 1_{L}\right\}$. In this case, $\mathrm{N}_{2}$ is a strong $L$-negation with three equilibrium points, namely $b, c$ and $d$.
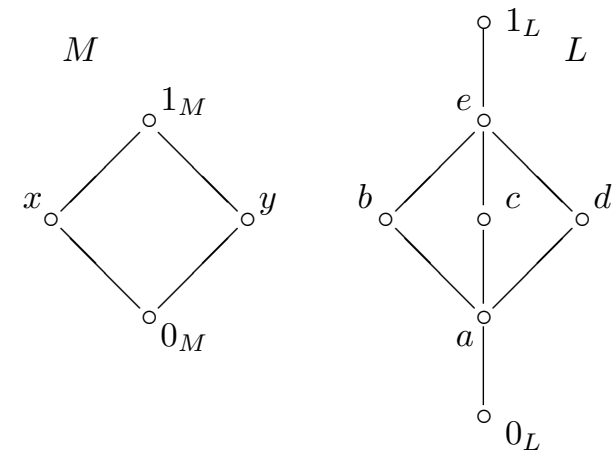

Figure 1: Hasse diagrams of lattices $M$ and $L$

Example 2.15 Consider the lattice $L_{0}$ obtained from lattice $L$ in Figure 1 by omitting the point a. Then, it does not exist a strong negation for this lattice. If $N$ is such negation, then we should consider $0_{L}<N(e)<N(b), N(c), N(d)<1_{L}$ which is not possible due to the injectivity of a strong negation. It is important to point out that the fact we can't define a strong negation over a lattice in general, as shown in this example, is not due to the fact that the order considered is partial, nor it is due to the fact that the lattice is discrete. It is easy to see that it is not possible to define a strong negation on the linear ordered lattice $L=\{0,1\} \cup[2,3]$ as well, considering the usual linear order of real numbers $\leqslant$ on $L$.

Proposition 2.16 Let $N: L \rightarrow L$ be a function, $\rho$ be an L-automorphism and $i \in\{1,2,3,4,5\}$. $N$ satisfies $(\mathrm{Ni})$ if and only if $N^{\rho}$ satisfies (Ni). Moreover, $e$ is an equilibrium point of $N$ if and only if $\rho^{-1}(e)$ is an equilibrium point of $N^{\rho}$.

Proof: Suppose $N$ satisfies $(N i)$ with $i \in$ $\{1,2,3,4\}$, then

(N1) $N^{\rho}\left(0_{L}\right)=\rho^{-1}\left(N\left(\rho\left(0_{L}\right)\right)\right)=\rho^{-1}\left(N\left(0_{L}\right)\right)=$ $\rho^{-1}\left(1_{L}\right)=1_{L}$. Analogously it can be proved that $N^{\rho}\left(1_{L}\right)=0_{L}$

(N2) If $x \leqslant_{L} \quad y$ then $\rho(x) \leqslant_{L} \quad \rho(y)$ and hence $N(\rho(y)) \leqslant_{L} N(\rho(x))$. Therefore, by isotonicity of $\rho^{-1}, \rho^{-1}\left(N(\rho(y)) \leqslant{ }_{L} \rho^{-1}(N(\rho(x)))\right.$;

(N3) $N^{\rho}\left(N^{\rho}(x)\right)=\rho^{-1}\left(N\left(\rho\left(\rho^{-1}(N(\rho(x)))\right)\right)\right)=$ $\rho^{-1}(N(N(\rho(x))))=\rho^{-1}(\rho(x))=x$;

(N4) Straightforward;

(N5) If $N^{\rho}(x) \in\left\{0_{L}, 1_{L}\right\}$ then, by eq. (2) and considering that $\rho^{-1}\left(0_{L}\right)=0_{L}$ and $\rho^{-1}\left(1_{L}\right)=1_{L}$ we have $N(\rho(x)) \in\left\{0_{L}, 1_{L}\right\}$. Thus, by (N5), $\rho(x) \in$ $\left\{0_{L}, 1_{L}\right\}$ which implies that $x=0_{L}$ or $x=1_{L}$;

If $N(e)=e$ then $N\left(\rho\left(\rho^{-1}(e)\right)\right)=e$ and hence $N^{\rho}\left(\rho^{-1}(e)\right)=\rho^{-1}\left(N\left(\rho\left(\rho^{-1}(e)\right)\right)\right)=\rho^{-1}(e)$.

Reciprocal is straightforward from the previous item and the fact that for any function $f: L \rightarrow L$, $\left(f^{\rho}\right)^{\rho^{-1}}=f$.

Corollary 2.17 Let $N: L \rightarrow L$ be a function and $\rho$ be an L-automorphism. $N$ is an (strong, strict, 
frontier) L-negation if and only if $N^{\rho}$ is an (strong, strict, frontier) L-negation.

\subsection{Equivalence Functions}

In image processing equivalences are usually considered to make a comparison between two images. One of the most common definition of equivalence was proposed by Fodor and Roubens in [22].

Definition 2.18 Let $L$ be a bounded lattice. A function $E F: L^{2} \rightarrow L$ is called an L-equivalence if it satisfies the following conditions:

(F1) $E F(x, y)=E F(y, x)$ for all $x, y \in L$;

$(F 2) E F\left(0_{L}, 1_{L}\right)=E F\left(1_{L}, 0_{L}\right)=0_{L}$;

(F3) $E F(x, x)=1_{L}$ for all $x \in L$;

(F4) If $x \leqslant_{L} y \leqslant_{L} z$ then $E F(x, z) \leqslant_{L} E F(x, y)$.

Nonetheless, equivalence functions as those described in Definition 2.18 do not allow us to ensure that only $\left(0_{L}, 1_{L}\right)$ and $\left(1_{L}, 0_{L}\right)$ are assigned to $0_{L}$, i.e., it could exist a pair $(x, y) \in L \backslash\left\{0_{L}, 1_{L}\right\}$ such that $E F(x, y)=0_{L}$. This is a disadvantage for comparing images since it cannot be ensured that two images in black and white are opposite if and only if one is the negative of the other. In order to solve this problem among others, Bustince et al. in [11] redefined (on $[0,1]$ ) Fodor and Roubens equivalences by adding some constraints and introducing the concept of restricted equivalence function. A generalization for bounded lattices is given in the following definition.

Definition 2.19 Let $N$ be a strict negation on $L$. A function REF : $L^{2} \rightarrow L$ is called a restricted equivalence function on $L$ with respect to $N$, or just an $L-R E F$ with respect to $N$, if it satisfies, for all $x, y, z \in L$, the following conditions:

(L1) $R E F(x, y)=R E F(y, x)$;

(L2) $\operatorname{REF}(x, y)=1_{L}$ if and only if $x=y$;

(L3) $\operatorname{REF}(x, y)=0_{L}$ if and only if $x=1_{L}$ and $y=0_{L}$, or $x=0_{L}$ and $y=1_{L}$;

(L4) $R E F(x, y)=R E F(N(x), N(y))$;

$(L 5)$ if $x \leqslant{ }_{L} y \leqslant_{L} z$ then $\operatorname{REF}(x, z) \leqslant{ }_{L}$ $\operatorname{REF}(x, y)$.

It is noteworthy that in the original version of restricted equivalence functions (see Definition 7 in [11]), the negation $N$ must be strong since, for many applications, specially in image processing, $(L 4)$ it ensures that a given property is preserved when the negative of an image instead of the image itself is considered. In general, though, it is not necessary to exist a strong negation for a given lattice $L$. This fact has led us to consider strict negations since this kind of negation always exists for non-trivial bounded lattices, as displayed in Example 2.13. On the other hand, the requirement of $N$ as a strict negation cannot be weakened, since a contradiction between $(L 3)$ and $(L 4)$ would otherwise arise.

\section{Extension Methods}

This section starts with the application of two different methods for extending fuzzy operators defined in previous works $[30,32]$. In [30] it was introduced an extension method via retractions that generalizes the extension proposed by Saminger-Platz et al. in [34]. In [32] we proposed a new extension method, called extension method via e-operators, which was aimed at achieving more efficiency to preserve the properties of extended operators.

\subsection{Extension via Retractions}

Let $M$ be a complete ordinary sublattice of $L$ and $T^{M}$ be a t-norm on $M$. Under these conditions, Saminger-Platz et al. in [34] have proposed a method for extending the t-norm $T^{M}$ from $M$ to $L$. They start by describing a way to extend this t-norm for $0_{L}$ and $1_{L}$ using the function

$T^{M \cup\left\{0_{L}, 1_{L}\right\}}(x, y)=\left\{\begin{aligned} x \wedge_{L} y, & \text { if } 1_{L} \in\{x, y\} ; \\ 0_{L}, & \text { if } 0_{L} \in\{x, y\} \\ T^{M}(x, y), & \text { otherwise. }\end{aligned}\right.$

Then, considering a function that takes an element $x$ belonging to $L$ and assigns to it $x^{*}=$ $\sup _{M}\left\{z \mid z \leqslant_{L} x, z \in M \cup\left\{0_{L}, 1_{L}\right\}\right\}$ it is proved that the t-norm given by

$W_{T^{M}}^{L}(x, y)=\left\{\begin{aligned} x \wedge_{L} y, & \text { if } 1_{L} \in\{x, y\} \\ T^{M \cup\left\{0_{L}, 1_{L}\right\}}\left(x^{*}, y^{*}\right), & \text { otherwise. }\end{aligned}\right.$

is an extension of $T^{M}$ from $M$ to $L$, i.e. the restriction of $W_{T^{M}}^{L}$ to $M$ is equal to $T^{M}$.

Note that this is a natural but drastic and particular way to extend t-norms since it collapses all elements of $L \backslash M$ on $M$ and only considers complete sublattices.

Seeking to propose a more general and flexible extension method, Palmeira and Bedregal presented in $[30,31,33]$ another way to extend t-norms, t-conorms and fuzzy negations considering $(r, s)$ sublattices. This method generalizes (4).

Proposition 3.1 [30] Let $M<L$ with respect to $(r, s)$. If $T$ is a t-norm on $M$ then $T^{E}: L \times L \longrightarrow L$ defined by

$$
T^{E}(x, y)=\left\{\begin{aligned}
x \wedge_{L} y, & \text { if } 1_{L} \in\{x, y\} \\
s(T(r(x), r(y))), & \text { otherwise. }
\end{aligned}\right.
$$

is a t-norm which extends $T$ from $M$ to $L$.

In a similar way, it is possible to extend t-conorms (see Proposition 4.1 in [30]). For negations we have 
Proposition 3.2 [30] Let $M$ be a $(r, s)$-sublattice of $L$ and $N: M \longrightarrow M$ be a fuzzy negation. Then $N^{E}(x)=s(N(r(x)))$ for each $x \in L$ is a fuzzy negation that extends $N$ from $M$ to $L$.

It is worth noting that, in Proposition 3.1, $r$ must be a lower retraction, whereas in Proposition 3.2, it is arbitrary (i.e., it could be lower, upper or neither). This fact shows negations can be extended in a more flexible way.

An advantage of this extension method is that it can be applied for several operators in the framework of fuzzy logic and applications. We have investigated the behavior of this method for t-subnorms, lattice automorphisms and some other particular classes of fuzzy operators (see [31, 33]).

Now we are interested in applying this method to extend equivalence functions motivated by the fact that images can be represented by lattices. For instance, imagine you have an equivalence function $E$ that provides a good comparison between images $\mathrm{A}$ and B. If you consider both images as parts of a bigger one and if you want to make a new comparison, then you should have a function $E^{\prime}$ to make it. One possible solution is to consider an extension $E$ preserving its properties. Accordingly, our extension method can be a suitable alternative.

Theorem 3.3 Let $M$ be a $(r, s)$-sublattice of $L$ and $E F: M^{2} \rightarrow M$ an equivalence function. Then the function

$$
E F^{E}(x, y)=s(E F(r(x), r(y)))
$$

for each pair $(x, y) \in L^{2}$ is an equivalence function that extends $E F$ from $M$ to $L$.

Proof: It is clear that $E F^{E}$ satisfies $(F 1)$ since $E F$ is an equivalence function. Moreover, for all $x \in L$ we have

$$
E F^{E}(x, x)=s(E F(r(x), r(x)))=s\left(1_{M}\right)=1_{L}
$$

and

$$
E F^{E}\left(0_{L}, 1_{L}\right)=s\left(E F\left(r\left(0_{L}\right), r\left(1_{L}\right)\right)\right)=s\left(0_{M}\right)=0_{L}
$$

Analogously, one can prove that $E F^{E}\left(1_{L}, 0_{L}\right)=0_{L}$. Thus, $(F 2)$ and $(F 3)$ hold.

It remains to prove $(F 4)$. To do so, note that for all $x, y, z \in L$ such that $x \leqslant L \quad y \leqslant_{L} \quad z$ it follows that $r(x) \leqslant_{M} \quad r(y) \leqslant_{M} \quad r(z)$ and hence $E F(r(x), r(z)) \leqslant_{M} E F(r(x), r(y))$. Thus

$$
\begin{aligned}
& E F^{E}(x, z)=s(E F(r(x), r(z))) \\
& \leqslant{ }_{L} \quad s(E F(r(x), r(y))) \\
& =E F^{E}(x, y) \text {. }
\end{aligned}
$$

Notice that Definition 2.19 refines Definition 2.18, since it imposes some new constraints. Aside from property $(L 4)$, Definition 2.19 also requires that the unique elements assigned to $0_{L}$ by $R E F$ are $\left(0_{L}, 1_{L}\right)$ and $\left(1_{L}, 0_{L}\right)$ and that $R E F$ is evaluated as $1_{L}$ only for pairs with the same value in both coordinates. Thus, it reveals that we cannot extend restricted equivalence functions using the method provided by Propositions 3.1 and 3.2 .

If $M$ is a $(r, s)$-sublattice of $L, N$ is a fuzzy negation on $M$ and $R E F: M^{2} \rightarrow M$ a restricted equivalence function with respect to $N$ then the function $R E F^{E}: L^{2} \rightarrow L$ given by $R E F^{E}(x, y)=s(R E F(r(x), r(y)))$ satisfies property $(L 4)$

$$
\begin{aligned}
& R E F^{E}\left(N^{E}(x), N^{E}(y)\right) \\
& =s\left(R E F\left(r\left(N^{E}(x)\right), r\left(N^{E}(y)\right)\right)\right) \\
& =s(R E F(r(s(N(r(x)))), r(s(N(r(y)))))) \\
& =s(R E F(N(r(x)), N(r(y)))) \\
& =s(R E F(r(x), r(y))) \\
& =\operatorname{REF}(x, y)
\end{aligned}
$$

Moreover, if $r$ is such that $r(x)=0_{L}$ if and only if $x=0_{L}$ and $r(x)=1_{L}$ if and only if $x=1_{L}$ then supposing $\operatorname{REF}^{E}(x, y)=0_{L}$ we have that $s(R E F(r(x), r(y)))=0_{L}$. Since $s$ is an injective function it follows that $\operatorname{REF}(r(x), r(y))=0_{M}$ and hence $r(x)=1_{M}$ and $r(y)=0_{M}$ or $r(x)=0_{M}$ and $r(y)=1_{M}$ by (L3), what allows us to conclude that $x=1_{L}$ and $y=0_{L}$ or $x=0_{L}$ and $y=1_{L}$, i.e. $R E F^{E}$ satisfies property ( $\left.L 3\right)$ (the necessity side of (L3) is shown in Theorem 3.3). Nevertheless $R E F^{E}$ does not satisfy $(L 2)$, in general, as the following examples shows.

Example 3.4 Let $M$ and $L$ be the bounded lattices depicted in Figure 1. Then function $s: M \rightarrow L$ defined by $s\left(0_{M}\right)=0_{L}, s(x)=b, s(y)=d$ and $s\left(1_{M}\right)=1_{L}$ is a pseudo-inverse of the retraction

$$
r(t)=\left\{\begin{array}{cl}
0_{M}, & t=0_{L} ; \\
1_{M}, & t=1_{L} ; \\
y, & t=d ; \\
x, & \text { otherwise }
\end{array}\right.
$$

and hence $M$ is a $(r, s)$-sublattice of $L$. Then, $\operatorname{REF}^{E}(e, c)=s(\operatorname{REF}(r(e), r(c)))=$ $s(R E F(x, x))=s\left(1_{M}\right)=1_{L}$ showing that $R E F^{E}$ does not satisfies (L2).

The above discussion allows us to enunciate the following proposition (extension of a weak version of restricted equivalence functions).

Proposition 3.5 Let $M$ be a $(r, s)$-sublattice of $L$ and $R E F: M^{2} \rightarrow M$ a restricted equivalence function. Then, $R E F^{E}: L^{2} \rightarrow L$ given by $R E F^{E}(x, y)=s(R E F(r(x), r(y)))$ for all $x, y \in L$ satisfies

(L1) $R E F^{E}(x, y)=R E F^{E}(y, x)$;

(WL2) $\operatorname{REF}^{E}(x, x)=1_{L}$;

(L4) $R E F^{E}(x, y)=R E F^{E}\left(N^{E}(x), N^{E}(y)\right)$, being $N$ a fuzzy negation on $M$; 
$(L 5)$ if $x \leqslant_{L} \quad y \leqslant_{L} \quad z$ then $\operatorname{REF}^{E}(x, z) \leqslant_{L}$ $\operatorname{REF}^{E}(x, y)$.

Moreover, if $r(x)=0_{M}$ iff $x=0_{L}$ and $r(x)=1_{M}$ iff $x=1_{L}$ then

(L3) $\operatorname{REF}^{E}(x, y)=0_{L}$ if and only if $x=1_{L}$ and $y=0_{L}$ or $x=0_{L}$ and $y=1_{L}$.

Definition 3.6 Let $L$ be an arbitrary bounded lattice. A function $R E F: L^{2} \rightarrow L$ satisfying properties (L1), (WL2), (L3), (L4) and (L5) is called a weak restricted equivalence function.

\subsection{Extension via $e$-operators}

As we have seen in the previous subsection, the extension method proposed in [30] is not good enough to extend restricted equivalence functions. Similar problems arise when we try to verify which properties of fuzzy connectives are preserved by extension via retractions. For instance, Archimedian t-norms, as well as strong fuzzy negations are not preserved.

Seeking to develop a extension method more efficient in preserving the properties we have provided in [32] a method based in the idea of interval constructor using e-operator.

Definition 3.7 Let $M \unlhd L$ with respect to $\left(r_{1}, r_{2}, s\right)$. A mapping $\odot: M \times M \longrightarrow L$ is called an e-operator on $M$ if it is isotonic and satisfies, for each $a, b \in M$ and for each $x \in L$, the following conditions:

$$
r_{1}(a \odot b)=a \wedge_{M} b \text { and } r_{2}(a \odot b)=a \vee_{M} b
$$

$$
r_{1}(x) \odot r_{2}(x)=x
$$

In other words, if $M \unlhd L$ with respect to $\left(r_{1}, r_{2}, s\right)$ (by Definition 2.11 , there are two retractions $r_{1}, r_{2}: L \longrightarrow M$ with the same pseudo-inverse $s: M \longrightarrow L$ such that $\left.s \circ r_{1} \leqslant i d_{L} \leqslant s \circ r_{2}\right)$ the $e$-operator $\odot$ describes an isotonic way to relate retractions $r_{1}$ and $r_{2}$ with the meet and join operators of $M$, respectively, by (7).

Example 3.8 Given a bounded lattice $\left\langle L, \wedge_{L}, \vee_{L}, 0_{L}, 1_{L}\right\rangle$, it is possible to define other bounded lattice $\langle\mathbb{L}, \wedge, \vee,[0,0],[1,1]\rangle$, named interval lattice (see [4]) where

$$
\mathbb{L}=\left\{[x, y]: x, y \in L \text { and } x \leqslant_{L} y\right\}
$$

and its operations are given by

$$
[x, y] \wedge[a, b]=\left[x \wedge_{L} a, y \wedge_{L} b\right]
$$

and

$$
[x, y] \vee[a, b]=\left[x \vee_{L} a, y \vee_{L} b\right] .
$$

Naturally, $L$ can be seen as a $\left(r_{1}, r_{2}, s\right)$-sublattice of $\mathbb{L}$ by considering the lower retraction $r_{1}: \mathbb{L} \longrightarrow L$ given by $r_{1}([x, y])=x$ with pseudo-inverse $s(x)=$ $[x, x]$, for all $x \in L$. The function $r_{2}([x, y])=y$ is an upper retraction from $\mathbb{L}$ to $L$ with the same $s$ as pseudo-inverse. It is easy to check that $s \circ r_{1} \leqslant i d \leqslant$ $s \circ r_{2}$. Therefore, $L \unlhd \mathbb{L}$ with respect to $\left(r_{1}, r_{2}, s\right)$.

Thus, by Definition 3.7, the mapping $\odot: L \times L \longrightarrow$ $\mathbb{L}$ defined by

$$
a \odot b=\left[a \wedge_{L} b, a \vee_{L} b\right]
$$

for each $a, b \in L$, is trivially an e-operator on $L$.

\section{Restricted Dissimilarity Function}

According to the literature, there are different ways to define functions to measure similarity or dissimilarity between two images. Bustince et al. [14] provided the notion of restricted dissimilarity functions based on error metric for images proposed by [3].

Definition 4.1 Let $L$ be a bounded lattice. A function $d_{L}: L^{2} \rightarrow L$ is called a restricted dissimilarity function on $L$ (for short $L-R D F$ ) if it satisfies, for all $x, y, z \in L$, the following conditions:

(D1) $d_{L}(x, y)=d_{L}(y, x)$;

(D2) $d_{L}(x, y)=1_{L}$ if and only if either $x=1_{L}$ and $y=0_{L}$ or $x=0_{L}$ and $y=1_{L}$;

(D3) $d_{L}(x, y)=0_{L}$ if and only if $x=y$;

$(D 4)$ if $x \leqslant_{L} y \leqslant_{L} z$ then $d_{L}(x, y) \leqslant_{L} d_{L}(x, z)$ and $d_{L}(y, z) \leqslant{ }_{L} d_{L}(x, z)$.

As we have made for restricted equivalence functions, here we apply both extension methods via retractions and via e-operators to extend restricted dissimilarity functions.

Proposition 4.2 Let $M$ be a $(r, s)$-sublattice of $L$ and suppose $r$ is such that $r(x)=0_{M}$ if and only if $x=0_{L}$ and $r(x)=1_{M}$ if and only if $x=1_{L}$. If $d_{M}: M^{2} \rightarrow M$ a restricted dissimilarity function, then $d_{M}^{E}(x, y)=s\left(d_{M}(r(x), r(y))\right)$ for each $x, y \in L$ satisfies (D1), (D2) and (D4). Moreover, $d_{L}(x, x)=0_{L}$ for every $x \in L$.

Proof: From Proposition 3.5, it is clear that $(D 1)$ holds. Moreover, for every $x \in L$ it follows that $d_{M}^{E}(x, x)=s\left(d_{M}(r(x), r(x))\right)=s\left(0_{M}\right)=0_{L}$. So, it remains to prove $(D 2)$ and $(D 4)$.

Supposing $d_{M}^{E}(x, y)=1_{L}$ then we have that $s\left(d_{M}(r(x), r(y))\right)=1_{L}$ which implies $d_{M}(r(x), r(y))=r\left(1_{L}\right)=1_{M}$ and hence either $r(x)=1_{M}$ and $r(y)=0_{M}$ or $r(x)=0_{M}$ and $r(y)=1_{M}$. Since $r(x)=0_{M}$ if and only if $x=0_{L}$ and $r(x)=1_{M}$ if and only if $x=1_{L}$ then either $x=1_{L}$ and $y=0_{L}$ or $x=0_{L}$ and $y=1_{L}$. On the other hand, $d_{M}^{E}\left(1_{L}, 0_{L}\right)=s\left(d_{M}\left(r\left(1_{L}\right), r\left(0_{M}\right)\right)\right)=$ $s\left(d_{M}\left(1_{M}, 0_{M}\right)\right)=s\left(1_{M}\right)=1_{L}$. By item 1 it holds that $d_{M}^{E}\left(0_{L}, 1_{L}\right)=0_{L}$. Therefore, $d_{M}^{E}$ satisfies (D2).

Now, take $x, y, z \in L$ such that $x \leqslant_{L} y \leqslant_{L} z$. In this case, we have that $r(x) \leqslant_{M} r(y) \leqslant_{M} r(z)$ and 
hence $d_{M}(x, y) \leqslant_{L} d_{M}(x, z)$. Then

$$
\begin{array}{rll}
d_{M}^{E}(x, y) & = & s\left(d_{M}(r(x), r(y))\right) \\
& \leqslant & s\left(d_{M}(r(x), r(z))\right) \\
= & d_{M}^{E}(x, z)
\end{array}
$$

Analogously, one can prove that $d_{M}^{E}(y, z) \leqslant L$ $d_{M}^{E}(x, z)$, so $(D 4)$ holds.

In other words, Proposition 4.2 says that the extension method via retractions is not efficient to extend restricted dissimilarity functions, since only a weak version of this kind of function is recovered. The same happened for the extension of restricted equivalence functions with this method, as seen in Section 3.1. Using the extension method via e-operators, though, makes possible to extend restricted dissimilarity functions successfully.

Proposition 4.3 Let $M \unlhd L$ with respect to $\left(r_{1}, r_{2}, s\right), \odot: M \times M \rightarrow L$ be an e-operator and $d_{M}: M^{2} \rightarrow M$ a restricted dissimilarity function. Then the function $\left(d_{M}\right) \stackrel{E}{\odot}: L^{2} \rightarrow L$ given by

$$
\left(d_{M}\right)_{\odot}^{E}(x, y)=d_{M}\left(r_{1}(x), r_{1}(y)\right) \odot d_{M}\left(r_{2}(x), r_{2}(y)\right)
$$

for all $x, y \in L$, is a restricted dissimilarity function on $L$.

Proof: Similar to proof of Theorem 4.14.

The following theorem proposes a way to construct restricted dissimilarity functions from restricted equivalence functions.

Theorem 4.4 Let REF : $L^{2} \rightarrow L$ be an $L-R E F$ with respect to $N$. If $N^{\prime}$ a frontier $L$-negation (not necessarily equal to $N$ ) then, the function defined by

$$
d_{L}(x, y)=N^{\prime}(R E F(x, y)) \text { for all } x, y \in L
$$

is a restricted dissimilarity function.

Proof: Analogously to the proof of Theorem 5 in $[12]$.

Corollary 4.5 Let $M \unlhd L$ with respect to $\left(r_{1}, r_{2}, s\right)$, $\odot: M \times M \rightarrow L$ be an e-operator and $R E F$ : $M^{2} \rightarrow M$ a restricted equivalence function. If $N$ is a fuzzy negation on $M$, then $d_{L}: L^{2} \rightarrow L$ given by $d_{L}(x, y)=N_{\odot}^{E}\left(R E F_{\odot}^{E}(x, y)\right)$ for all $x, y \in L$, is a restricted dissimilarity function.

Proof: Straightforward.

\section{Final Remarks}

This work has addressed two different methods (via retractions EMR and via e-operators EMP) to extend restricted equivalence functions, restricted dissimilarity functions.
As observed in previous works $[31,32,33]$, results have shown that EMP method is more efficient in preserving properties of extended operators whereas, EMR method is more robust than EMP when the minimality of the extension is considered.

For future works, we wish to study the behavior of the extension of other important functions related to image processing such as penalty, aggregations and overlaps functions.

\section{References}

[1] M. Baczyński. Residual implications revised. notes on the Smets-Magrez theorem. Fuzzy Sets and Systems, 145(2):267-277, 2004.

[2] M. Baczyński and B. Jayaram. Fuzzy Implications. Springer, Berlin-Heidelberg, 2008.

[3] A.J. Baddeley. An error metric for images. Robust Computer Vision. Wichmann, Karlsruhe, pp. 59-78, 1992.

[4] B. Bedregal. On interval fuzzy negations. Fuzzy Sets and Systems, 161: 2290-2313, 2010.

[5] B. C. Bedregal, G. Beliakov, H. Bustince, J. Fernandez, A. Pradera and R. Reiser. (S,N)Implications on bounded lattices. M. Baczyński et al. (eds), Advances in Fuzzy Implication Functions. Studies in Fuzziness and Soft Computing, Springer, Berlin 2013.

[6] B. Bedregal, G. Beliakov, H. Bustince, J. Fernandez, A. Pradera and R. Reiser. Negations generated by bounded lattices t-norms. 14 th Internacional Conference on Information Processing and Management of Uncertainty in Knowledge-based Systems, Catania, Italy, 2012.

[7] B.C. Bedregal, H.S. Santos, and R. CallejasBedregal. T-norms on bounded lattices: tnorm morphisms and operators. In Proceedings of 2006 IEEE International Conference on Fuzzy Systems, pages 22-28, Vancouver, Canada, 2006.

[8] B. C. Bedregal and A. Takahashi. The best interval representations of t-norms and automorphisms. Fuzzy Sets and Systems, 157:3220-3230, 2006.

[9] G. Birkhoff. Lattice theory. American Mathematical Society, Providence. RI, 1973.

[10] M. Bóna. A walk through combinatorics. An introduction to enumeration and graph theory World Scientific Publishing, Singapore, 2006.

[11] H. Bustince, E. Barrenechea and M. Pagola. Restricted equivalence functions. Fuzzy Sets and Systems, 157: 2333-2346, 2006.

[12] H. Bustince, E. Barrenechea and M. Pagola. Relationship between restricted dissimilarity functions, restricted equivalence functions and normal $E_{N}$-functions: Image thresholding invariant. Pattern Recognition Letters, 29: 525536, 2008.

[13] H. Bustince, P. Burillo, and F. Soria. Automorphisms, negations and implication oper- 
ators. Fuzzy Sets and Systems, 134(2):209-229, 2003.

[14] H. Bustince, J. Fernandez, R. Mesiar, A. Pradera and G. Beliakov. Restricted dissimilarity functions and penalty functions. EUSFLAT-LFA 2011, Aix-les-Bains, France, 2011.

[15] H. Bustince, J. Fernandez, A. Kolesárová and R. Mesiar. Generation of linear orders for intervals by means of aggregation functions. Fuzzy Sets and Systems, 220: 69-77, 2013.

[16] H. Bustince, M. Pagola and E. Barrenechea. Definition and construction of fuzzy DIsubsethood measures: Application to the global comparison of images. Information Science, 117(3): 906-929, 2007.

[17] S. Burris and H. P. Sankappanavar. A course in universal algebra. The Millennium Edition, New York 2005.

[18] T. Calvo. On mixed De Morgan triplets. Fuzzy Sets and Systems, 50:47-50, 1992.

[19] T. Chaira and A.K. Ray. Region extraction using fuzzy similarity measures. J. Fuzzy Math., 11(3): 601-607, 2003.

[20] T. Chaira and A.K. Ray. Fuzzy measures for color image retrieval. Fuzzy Sets and Systems, 150: 545-560, 2005.

[21] B.A. Davey and H.A. Priestley. Introduction to lattices and order. Cambridge University Press, Cambridge, 2nd edition, 2002.

[22] J. Fodor and M. Roubens. Fuzzy preference modelling and multicriteria decision support. Kluwer Academic Publisher, Dordrecht, 1994.

[23] J. Goguen. L-fuzzy sets. Journal of Mathematical Analysis and Applications, 18(1):145-174, 1967.

[24] G. Grätzer. Lattice theory: First concepts and distributive lattices. Dover Publications, 2009.

[25] G. Grätzer. Lattice theory: Foundation. Birkhäuser, 2011.

[26] T. W. Hungerford. Algebra. Graduate texts in mathematics, Springer-Verlag, 2000.

[27] A. Jurio, M. Pagola, D. Paternain, C. LopezMolina and P. Melo-Pinto. Interval-valued restricted equivalence functions applied on clustering techniques. IFSA-EUSFLAT, 2009.

[28] C. Lopez-Molina, B. De Baets and H. Bustince. Generating fuzzy edge images from gradient magnitudes. Computer Vision and Image Understanding, 115(11):1571-1580, 2011.

[29] M. Mas, M. Monserrat, J. Torrens, and E. Trillas. A survey on fuzzy implication functions. IEEE Trans. on Fuzzy Systems, 15(6):11071121, 2007.

[30] E.S. Palmeira and B. Bedregal. Extension of fuzzy logic operators defined on bounded lattices via retractions. Computers and Mathematics with Applications, 63:1026-1038, 2012.

[31] E.S. Palmeira and B.C. Bedregal. Extension of t-subnorms on bounded lattices via retractions. 30th Annual Conference of the North American Fuzzy Information Processing Society. El Paso, TX, 2011.

[32] E.S. Palmeira, B. Bedregal and R. Mesiar. A new way to extend t-norms, t-conorms and negations. Fuzzy Sets and Systems, 240:1-21, 2014.

[33] E.S. Palmeira, B. Bedregal, J. Fernandez and A. Jurio. On extension of lattice-valued implications via retractions. Fuzzy Sets and Systems. 240:66-85, 2014.

[34] S. Saminger-Platz, E. P. Klement and R. Mesiar. On extensions of triangular norms on bounded lattices. Indagationes Mathematicae, 19(1): 135-150, 2008.

[35] D. Van der Weken, M. Nachtegael and E.E. Kerre. Using similarity measures and homogeneity for the comparison of images. Image and Vision Comput., 22:695-702, 2004. 